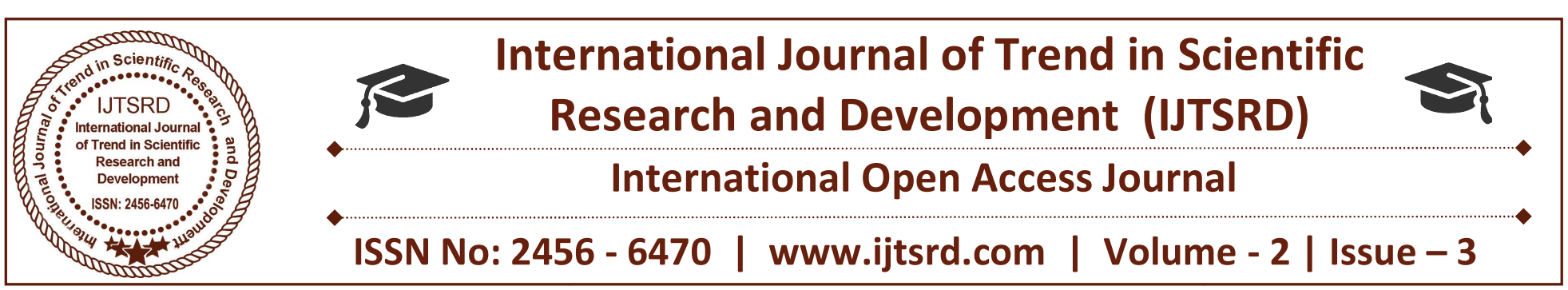

\title{
A Guarantee of Profit Maximization on Multi-Cloud Public Platform
}

\author{
Anita D. Khadka, Shreya S. Inamdar, Payal S. Vaidya, Prof. Sonali Patil \\ Department of Computer, SRTTC Faculty of Engineering, \\ Pune, Maharashtra, India
}

\begin{abstract}
\end{abstract}
An effective and efficient way to provide computing resources and services to clients on their demand, cloud computing is one the most popular field. From cloud service providers' perspective, profit is the most important consideration along with user-friendly quality services, and it is mainly determined by the configuration of a cloud service platform under given market demand. In any case, a single long-term renting utilized as a part of past modules can't ensure the service quality yet prompts major issue in storage and resource allocation. In this paper we have apportioned the capacity for customer ask for, if the capacity is accessible at that point ask for is acknowledged by cloud and in the event that it is occupied or capacity is unavailable then we allocate storage by selecting minimum cost using hill climbing algorithm. This paper also presents a proposed model for cloud computing scheduling based on multiple server. Cloud services are efficiently and optimally allocated to satisfy the requirements of customer. The result shows that our scheme can not only guarantee the service quality of all requests, but also obtain more profit than the latter. To enhance the quality services to the client, a tool is developed that will track the data usage and storage spaces of all the users and provide flexibility. At last, a progressions of calculations are conducted to look at the benefit of our proposed scheme with that of the single renting scheme.

Keywords: Cloud Computing, Guaranteed Service Quality, Multi Server System, Service Level Agreement, Scheduling Models, Waiting time.

\section{INTRODUCTION}

Cloud computing is the delivery of resources and computing as a service instead of a product and centralizes management of resources and services which delivers hosted services over the Internet. Customers use and pay for services on-demand whereas not considering the direct infrastructure prices and so the following maintenance worth. As a result of such benefits, cloud computing is turning into voluminous and has received respectable attention recently. Nowadays, there are several cloud service suppliers, like Amazon EC2, Aws, Layer shift, drive HQ etc. As a kind of recent IT industrial model, profit may be an important concern of cloud service suppliers. The cloud service suppliers rent resources from infrastructure suppliers to line up the service platforms and supply paid services to customers to make more profits. For cloud service suppliers, the simplest way to tack their cloud service platforms to induce the biggest profit becomes increasingly the most focus that they concentrate to. The most effective configuration draw back with profit maximization of cloud service suppliers has been researched in our previous researches that assumed that the cloud service demand is assumed ahead and not plagued by external factors. However, the request arrival rate of a service provider is plagued by many factors in actual, and consumer satisfaction is that the foremost important issue. As an example, customers would possibly submit their tasks to a cloud computing platform or execute them on their native computing platforms. The consumer behaviour depends on if the cloud service is attractive enough to them. To tack a cloud service platform properly, the cloud service provider got to acumen consumer satisfaction affects the service demands. 
Hence, considering consumer satisfaction in profit improvement drawback is vital.

However, few existing works take consumer satisfaction into thought to find profit maximization drawback, or the prevailing works considering consumer satisfaction do not provides an accurate formalized definition for it. To handle the matter, this paper adopts the thought in Business Administration, and first defines the consumer satisfaction level of cloud computing. Support the definition of consumer satisfaction and have a tendency to tend to create a profit maximization model inside that the impact of consumer satisfaction on quality of service (QoS) and prize of service $(\mathrm{PoS})$ is taken under consideration. From associate economic posture, a pair of factors moving consumer satisfaction area unit QoS and PoS. The PoS is determined by cloud service suppliers. The QoS is determined by the service capability of a cloud service provider that for the foremost half depends on its platform configuration below the given rating strategy. It is real because it improves the consumer satisfaction level, it push the QoS, which could be achieved by configuring cloud platform with higher service capability. Doing so will have a bearing on a cloud service supplier from a combine of a sides. On one hand, the upper shopper satisfaction level finally winds up inside ensuing market share, so the cloud service supplier will gain additional revenues. On the choice hand, additional resources unit rented to boost the service capability, that finally winds up inside the increase of prices. Hence, the last word answer of up profit is to appear out associate best cloud platform configuration theme. Throughout this paper, we have a tendency to form a consumer satisfaction aware profit optimisation model and propose a precise hill rising algorithm to appear out the numeric best cloud configuration for cloud service suppliers.

\section{Literature Survey}

1] A Profit Maximization Scheme with Guaranteed Quality of Service in Cloud Computing, Jing Mei, Kenli Li, Aijia Ouyang, Keqin Li, Vol. no. 64, 06 February 2015 [Citation information: DOI10.1109/TC.2015.2401021, IEEE Transactions on Computers]

Cloud computing centralizes management of resources and services and delivers hosted services over the Internet. The hardware, software, databases, information, and all resources are concentrated and provided to consumers on-demand. In this paper, aim at researching the multi-server configuration of a service provider such that its profit is maximized. The drawback of this paper is, in Traditional way, cannot guarantee the quality of all requests and also wastage a great amount of resources. So to overcome this problem we used scheme outperforms the SQU scheme in terms of both of service quality and profit. In this, we review recent works relevant to the profit of cloud service providers. Profit of specialist organizations is related with numerous variables, for example, the value, the market request, the framework configuration, the consumer loyalty. In this paper a profit maximization issue is defined and comprehended to get the ideal multi-server configuration which would product be able to more profit than the ideal configuration. this paper considered the profit maximization issue in a homogeneous cloud condition, in light of the fact that the investigation of a heterogenous domain is considerably more convoluted than that of a homogenous domain. ${ }^{[1]}$

2] Enhancing Cloud Computing Scheduling based on Queuing Models, Mohamed Eisa, E. I Esedimy, M.Z. Rashad, Volume 85 - No 2, January 2014

In recent years, cloud computing has developed from an beginning period answer for a standard operational model for venture applications. This paper goes for supporting exploration in this region by giving an overview of the best in class of QoS demonstrating approaches appropriate for cloud frameworks. In general clouds are deployed into three access levels: Software-as-a Service(SaaS), Platform-as-a-Service (PaaS) and Infrastructure-as-a-Service (IaaS). The drawback of this paper is it needs a lot of time of routing end user requests. So, to overcome this we used cloud computing algorithms based on parallel algorithms to decrease the time of routing end users' requests. In this paper, the proposed model based on queuing models. Routing incoming requests to the queue with the smallest workload reduced workload, response time and the average length of the queue.

3] Resource allocation strategies used in cloud computing: A critical analysis, Neeraj Kumar Pandey, Sumit Chaudhary, N.K. Joshi, 978-1-5090-32105/16/\$31.00 C2016 IEEE.

Cloud services are effectively and ideally designated to fulfill the necessities of client. The focal point of 
this paper is itemized and relative examination on different resource allocation strategies by preserving the service level agreement (SLA). There are many strategies are used to optimize the resources. All system is concentrating on the allotment of resource by thinking about the various parameters i.e. value, reliability, productivity, utility, fulfillment of client, adaptability and Quality of Service(QOS). The drawback of this paper is it is difficult to allocate resources when multiple jobs arrive. So, to overcome this type of problem we should provide services

pretending that the resources are at users' place. The paper likewise centers around the extent of

parameters used for comparison change in each technique followed in regard with resource allocation methodologies in cloud environment.

4] A cloud computing resource allocation model based on combinatorial double auction, Jun $\mathrm{Xu}$, DOI10.1109/ICISCE.2016.12.

We know that users and providers have different requirements and objectives in an investment market. This paper presents a new cloud computing resource allocation model based on combinatorial double auction mechanism (CDA-CCRA) for more effective resource utilization. The main advantages of the proposed model are summarized as follows: (1) it can fulfil both client and provider necessities, (2) it can reduce the normal time for resource distribution and evaluating, and (3) it can produce higher incomes. Less system performance, economic efficiency these are the drawbacks of this model. So, to overcome this CDA-CCRA model allocates resources well in cloud computing environment. Future research will expand the CDA-CCRA model to intuitionistic fluffy condition.

\section{Proposed System}

In this project we have implemented the allocation of resources to the client based on their scheme. Hence, a client is the primary member who request for his storage then his request is passed by the mediator to the ISP. BSP the mediator who provides client the different scheme with low to high cost. BSP will give all the information about the cloud and his plans. As BSP pass the client request to main service provider, ISP will provide service to client only if the provider is available with his storage, if it is busy providing the resource to other client then it will send message to BSP stating he is busy or space not available. BSP will then ask the client whether he wants to wait for the same cloud or switch to another cloud. After this acknowledgment from BSP client will take decision according to his needs. Once the client confirms his request, BSP will add his profit prize to the original cost of the cloud prize and then send the total prize to the client in the form of text message and in case if, the user gets rejected by the ISP then user have to rerequest for the storage. The BSP here uses hill climbing algorithm which work searching the least prize cloud from the multiple cloud provider and send request to a particular cloud. Finally, after paying for the cloud, the client will successfully upload his files. This is the main aim of this project where resources will not be wasted and maximum profit will be calculated.

\section{System Architecture}

Software design alludes to the anomalous state structures of an item system, the prepare of making such structures, and the documentation of these structures. These structures are required to reason about the item system. In this undertaking our guideline point is to get benefitted from the administrations that are open from the infrastructure provider. Here we have to provide some benefits to both the provider (BSP and ISP). The main aim of this project is to save resources and provide benefits to all the service provider. In system architecture we have shown the flow of our system. How it works and how it gets executed. In system architecture we have defined three modules Customer, BSP and ISP. The customer here will request for storage so that he can upload his data in different cloud. BSP the middle agent will show the renting scheme for the different cloud. BSP send request customers request to the ISP who is the main cloud then ISP will accept customers request if the provider have space or available at that time. if it is busy or doesn't exist. In the way customers need is fulfilled and all the request if satisfied. Here we improve the quality of service and guarantee the renting plan. 


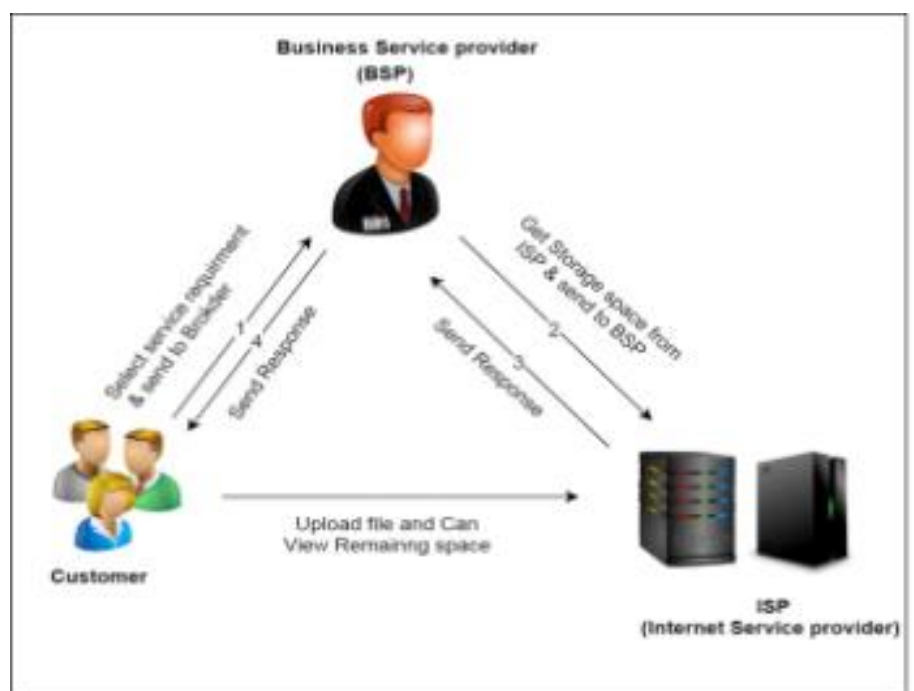

The system architecture consists of following modules:

\section{1) Customer:}

A customer will request for storage and pay according to his plans. He will invest to get the sources from multiple cloud.

\section{2) BSP:}

A Business Services Provider (BSP) is the messenger like a agent who carry every task of the customer and send them to the main cloud provider. BSP benefits from customers renting scheme. BSP share data from customer to main provider.

\section{3) ISP:}

An Infrastructure service provider(ISP) is a cloud service provider that delivers computer infrastructure on an outsourced basis to support enterprise operations. Typically, ISP provides storage, servers and data center space to customer on their request virtually.

\section{A. Advantages}

1) Performance is high.

2) Customer fulfillment in taking care of ideal setup issue with profit maximization

3) Improve the administration nature of cloud service provider.

4) Since the requests with holding up time $D$ are altogether assigned to brief servers, it is evident that all administration requests can ensure their due date and are charged in light of the workload as indicated by the SLA. Subsequently, the income of the service provider increases ${ }^{[1]}$.

5) Increase in the quality of service requests and maximize the profit of service providers ${ }^{[2]}$.

6) This plot joins short-term renting with long-term renting, which can lessen the asset squander extraordinarily and adjust to the dynamical request of processing limit ${ }^{[3]}$.

\section{B. Disadvantages}

1) In existing system, customer satisfaction did not give a proper formalized.

2) Optimal configuration problem with profit maximization in cloud service.

\section{Mathematical Model of System}

The mathematical model is illustrated properly that defines the problem statement of project, input data, output data and all the necessary constraints regarding project. Solution perspective for proposed scheme.

\section{A. Assumption}

IaaS provider, service providers are present in backend.

S - initial: Initially system will be in a state where no users are enrolled and as users are enrolled, system will change its state.

e- End state: [resource allocation]: The system will verify user on his request to access data base and system will declare the user as valid or invalid.

Fme: Queuing based model.

$\mathrm{X}$ : [requesting resource]: A customer submits its request to a service provider and pays for it based on the amount and the quality of the provided service.

Y: [allocating resource]: service provider will allocate the resource to one who have payed for the services.

DD: [Deterministic Data]: If requested space is available then the server will allocate that space to the client.

NDD: [Non-Deterministic Data]: If requested space is not available then it get rejected from the list.

$\mathrm{Mem}_{\text {shared: }}$ Cloud storage.

\section{Implementation}

In this project we have implemented various algorithm such as cloud computing, Multi-server module, Hill climbing module.

\section{A. Cloud Computing Model}

Cloud computing portrays a kind of outsourcing of computer administrations, like the manner by which the supply of power is outsourced. Clients can basically utilize it. They don't need to pressure where 
the power is from, how it is made, or transported. Consistently, they pay for what they expended. The thought behind cloud computing is comparable: The client can just utilize storage, registering power, or extraordinarily made advancement conditions, without worrying how this work inside. Cloud computing is normally Internet-based processing.

\section{B. Multi-server Model}

A cloud computing service provider serves users' service requests by using a multi-server system, which is constructed and maintained by an infrastructure vendor and rented by the service provider. The architecture detail of the multi-server system can be quite flexible. In this project, we consider the cloud service platform as a multi-server system with a service request queue.

\section{Customer module}

A customer submits a service request to a service provider which delivers services on demand. The customer receives the desired result from the service provider with certain service and pays for the service based on the amount of the service and the service quality.

\section{Business Service Providers module}

In this module the service providers considered as cloud brokers because they can play an important role in between cloud customers and infrastructure providers, and he can establish an indirect connection between cloud customer and infrastructure providers.

\section{E. Infrastructure Service Provider module}

In the three-tier structure, an infrastructure provider the basic hardware and software facilities. A service provider rents resources from infrastructure providers and prepares asset of services in the form of virtual machine(VM).

\section{F. Hill Climbing module}

Hill Climbing is a method to take care of certain optimization issues. In this procedure, we begin with an imperfect arrangement and the arrangement is enhanced over and over until the point when some condition is augmented. Starting with an imperfect arrangement is contrasted with beginning from the base of the slope, enhancing the arrangement is contrasted with strolling up the slope, lastly expanding some condition is contrasted with achieving the highest point of the hill.
(1) Algorithm

$>$ Evaluate the initial state.

$>$ Loop until the point that an answer is found or there are no new administrators left to be connected.

$>$ Select and apply another administrator

$>$ Evaluate the new state:

$>$ goal $\rightarrow$ quit better than current state $\rightarrow$ new current state

\section{Flow of Project}

1) Start

2) First, client will register on website.

3) 3.Client will send request to BSP for its requirement.

4) 4.BSP will accept client request and arrange according to its renting plan.

5) 5.Next, BSP will send the client request to ISP in queue format.

6) 6.BSP will check the status of ISP whether it is free or busy. if the ISP is free it will response to BSP and allocate the requested space according to the plans.

7) 7.If ISP is busy then it will put the request in queue or it will ask client to wait for its request or switch to another ISP.

8) 8.Space will be allocated to client as per renting plans.

9) 9.Finally, client will upload the data on website.

\section{Results}

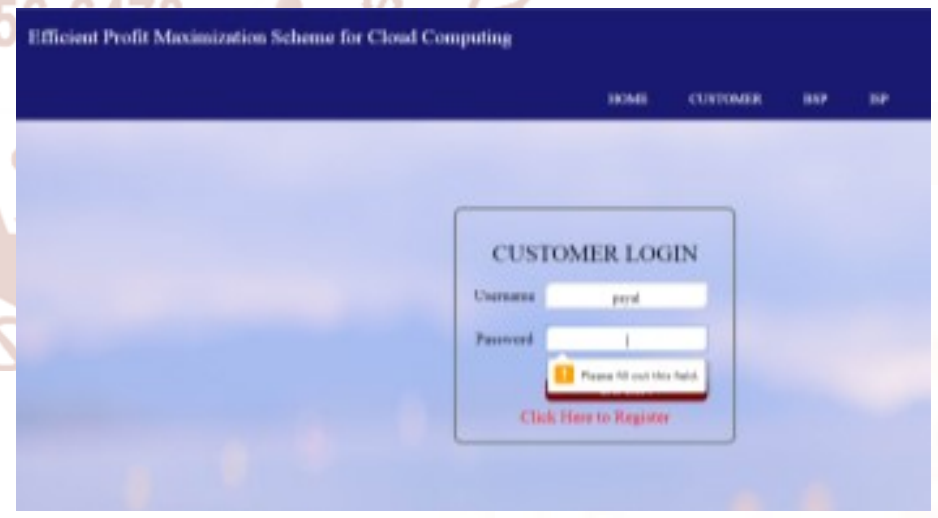

Fig. 1, Login form for different client 


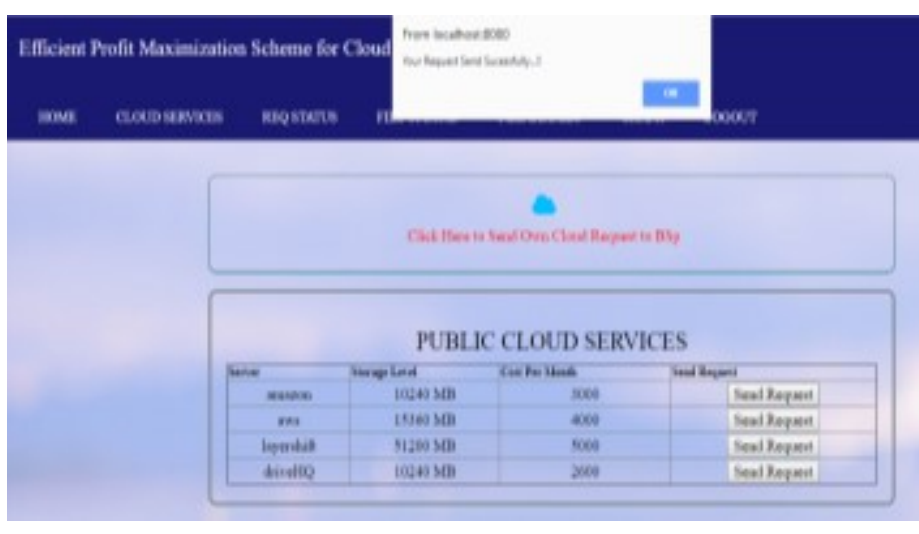

Fig. 2, Cloud Request

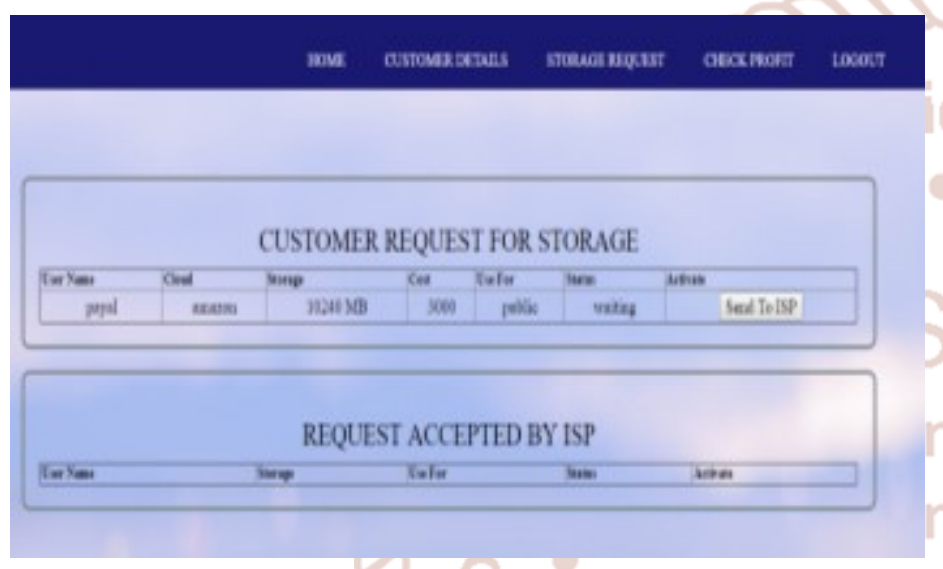

Fig. 3, Sending Request To BSP From ISP

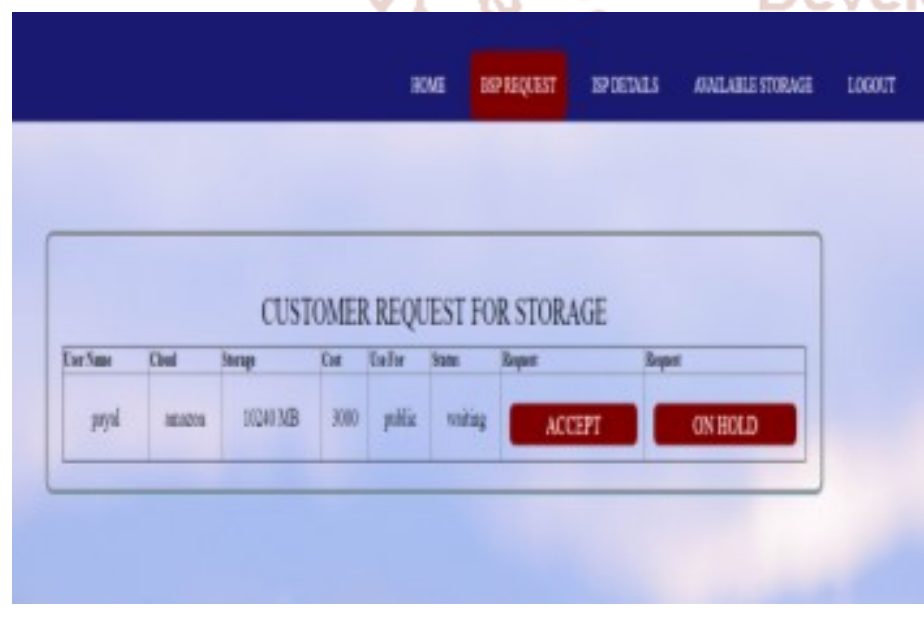

Fig. 4, Response Of ISP

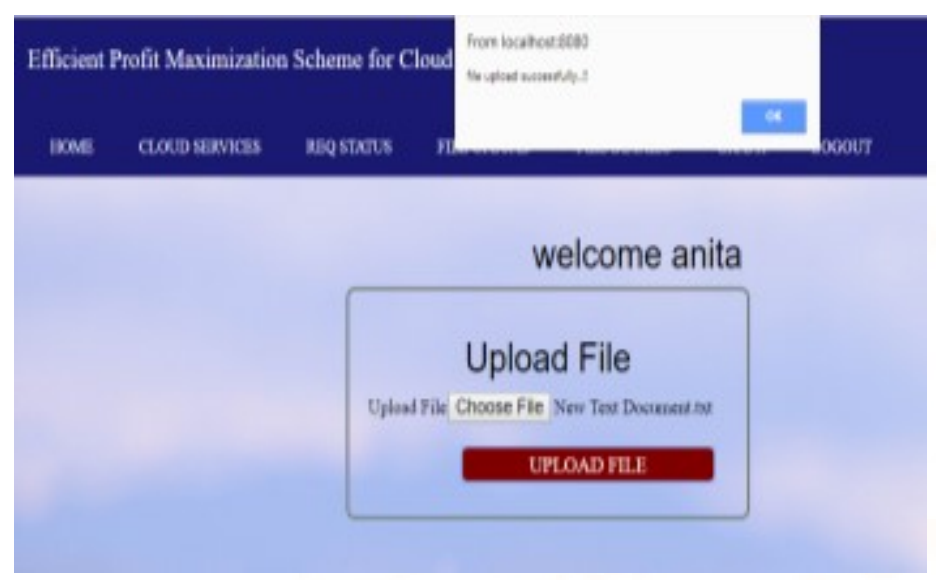

Fig. 5, File Upload Successfully

\section{Conclusion and future scope:}

In this project a brand-new approach referred to as resources allocation deals with methodology for service suppliers is projected to confirm the standard of service demands yet on enhance the profit and to reduce the wastage of resources, this methodology includes each the short term dealing methodology yet as long run dealing methodology. To vary the system sizes, a queuing model is enforced for multi-server system. The information hosting formula is enforced before the DQG methodology, to form the appropriate selection of cloud yet on enhance the profit of service supplier. After that, a difficulty of best configuration of profit increasing is outlined in these many aspects area unit determined. This projected double-dealing strategy is enforced to heterogeneous cloud setting. we have a tendency to use double dealing system so as to extend the profit of supplier. This project conjointly focuses on the scope of improvement in each strategy followed in regard with resource allocation methodologies in cloud setting. to reinforce the standard services to the consumer, a tool is to be developed that may track the information usage and storage areas of all the users and supply flexibility within the dealing to the consumer.

\section{Acknowledgement:}

The authors thank the anonymous reviewers for their valuable comments and suggestions. We would like to express our gratitude to all those who helped us to complete this work. We want to thank our internal and external guide for continuous help and generous assistance. They helped in a broad range of issues from giving us direction, helping to find the solutions, outlining the requirements and always having the time to see us. 
XI. References:

1) Jing Mei, Kenli Li, Aijia Ouyang, Keqin Li, “A Profit Maximization Scheme with Guaranteed Quality of Service in Cloud Computing", Vol. no. 64, 06 February 2015 [Citation information: DOI 10.1109/TC.2015.2401021, IEEE Transactions on Computers].

2) Mohamed Eisa, E. I. Esedimy, M. Z. Rashad, “ Enhancing Cloud Computing Scheduling based on Queuing", Volume 85 - No 2, January 2014.

3) Neeraj Kumar Pandey, Sumit Chaudhary, N.K. Joshi, "Resource allocation strategies used in cloud computing: A critical analysis", 978-15090-3210-5/16/\$31.00 (C) 2016 IEEE.

4) Jun $\mathrm{Xu}$, "A cloud computing resource allocation model based on combinatorial double auction", DOI 10.1109/ICISCE.2016.12.

5) Cloud computing Wikipedia.
6) http://en.wikipedia.org/wiki/Service level agreement.

7) K. Hwang, G. C. Fox, and J. J. Dongarra, Distributed and Cloud Computing, Morgan Kaufmann, 2012.

8) R. Buyya, D. Abramson, J. Giddy, and H. Stockinger, "Economic models for resource management and scheduling in grid computing," Concurrency and Computation: Practice and Experience, vol. 14, pp. 1507-1542, 2007.

9) R. Buyya, C. S. Yeo, S. Venugopal, J. Bromberg, and I. Brandic, "Cloud computing and emerging IT platforms: vision, hype, and reality for delivering computing as the 5th utility," Future Generation Computer Systems, vol. 25, no. 6, pp. 599-616, 2009.

10) Performability Models for Designing Disaster Tolerant Infrastructure-as-a-Service Cloud Computing Systems Bruno Silva, Paulo Maciel Center for Informatics (CIn) Federal University of Pernambuco (UFPE) Recife, Pernambuco. 